\title{
Endoscopic Versus Open Carpal Tunnel Release
}

\author{
Eon K. Shin ${ }^{1}$
}

Published online: 26 November 2019

(C) Springer Science+Business Media, LLC, part of Springer Nature 2019

\begin{abstract}
Purpose of Review Carpal tunnel syndrome is the most common compressive neuropathy encountered by hand and upper extremity surgeons. The predominant presentation includes symptomatic paresthesias in the median nerve distribution of the affected hand, frequently causing nocturnal disturbances. Surgical treatment requires division of the transverse carpal ligament, which can be performed through open and endoscopic means. Endoscopic techniques have evolved significantly since they were first introduced in the late 1980s. This manuscript reviews the literature to summarize the current state of carpal tunnel surgery. Recent Findings While endoscopic techniques have demonstrated superior early functional outcomes and a more rapid recovery, there are lingering concerns over the potential for nerve, vessel, and tendon injuries. These concerns have not been validated by the hand surgery literature, which ascribes similar rates of complications for both open and endoscopic surgical approaches. Moreover, patients report greater satisfaction with endoscopic surgical approaches compared with open techniques.

Summary In summary, the debate between proponents of open versus endoscopic carpal tunnel surgery continues. While surgeons who employ endoscopic techniques appear to be in the minority, there is a plethora of evidence to suggest that both approaches are comparably safe and equally effective.
\end{abstract}

Keywords Carpal tunnel syndrome $\cdot$ Endoscopic carpal tunnel surgery $\cdot$ Open carpal tunnel surgery $\cdot$ Return to work $\cdot$ Neuropraxia

\section{Introduction}

Carpal tunnel syndrome (CTS) is the most common peripheral nerve entrapment encountered by hand and upper extremity surgeons, accounting for over 600,000 surgeries in USA annually. Symptoms of CTS include numbness, pain, burning, weakness, and nocturnal paresthesias involving the median nerve distribution [1]. Conservative measures include splint immobilization and possibly corticosteroid injections for symptomatic relief. Surgery is indicated when conservative treatments have failed.

In 1933, Learmonth was the first to describe the surgical release of the transverse carpal ligament to treat posttraumatic median nerve compression [2]. In 1946, Cannon and Love [3]

This article is part of the Topical Collection on Compressive Neuropathies in the Upper Extremity

Eon K. Shin

ekshin@handcenters.com

1 Philadelphia Hand to Shoulder Center, Thomas Jefferson University Hospital, 1203 Langhorne-Newtown Road, Suite 335, Langhorne, PA 19047, USA then published their approach to patients suffering from nontraumatic compression of the median nerve. However, the concept of CTS was not popularized until Brain et al [4] expounded upon the pathophysiology of spontaneous nerve compression at the wrist and described the surgical release of the median nerve in six patients. In 1950 and over the subsequent decades, Phalen and others reported on numerous patients with idiopathic spontaneous CTS, treated by surgical transection of the transverse carpal ligament [5-10]. Open carpal tunnel release (OCTR) ultimately assumed the surgical standard of care in treating carpal tunnel syndrome.

Endoscopic surgery was not introduced until 1987 when Okutsu, a Japanese orthopedic surgeon, first reported division of the transverse carpal ligament in a patient with CTS using an endoscope [11]. In 1989, Chow reported the use of two incisions to transect the transverse carpal ligament endoscopically in the English language literature [12]. Following this introduction of endoscopic carpal tunnel release (ECTR) in USA, significant controversy ensued regarding the efficacy, safety, and success of these procedures. Numerous reports were published, describing technique modifications as well as results and complications. Among an early retrospective analysis of 61 ECTRs [13], results were rated as excellent in 
37 cases, good in 11, and poor in 4 . Nine patients were not available for follow-up evaluation. Decreased postoperative morbidity and a more rapid return to work were found with endoscopic surgery. However, one patient required repair of the median nerve. Another patient required a conventional carpal tunnel release 1 year after the first endoscopic surgery. The author concluded that the "results do not justify continued use of endoscopic approaches when conventional release is used as the standard for comparison."

While much of the controversy has waned over the use of endoscopic surgery, most hand and upper extremity surgeons still prefer open or mini-open approaches to treat CTS. According to a recent online questionnaire submitted to active members of the American Association for Hand Surgery, approximately $34 \%$ of respondents favored a standard open (extensile) incision, $46 \%$ favored a mini-open incision, and $20 \%$ preferred an endoscopic approach (Fig. 1) [14]. While surgeons agree that complete surgical release of the transverse carpal ligament should relieve CTS symptoms and improve function [15], the technique which benefits patients most is still contested.

For the purposes of this discussion, we will focus on a few quantified parameters in the hand surgery literature that have been used to compare open carpal tunnel surgery with endoscopic techniques. These parameters include: (1) return to work, (2) functional outcomes, (3) complications, and (4) cost. Unfortunately, much of the recent comparative data on this subject has been dominated by meta-analyses of existing case series.

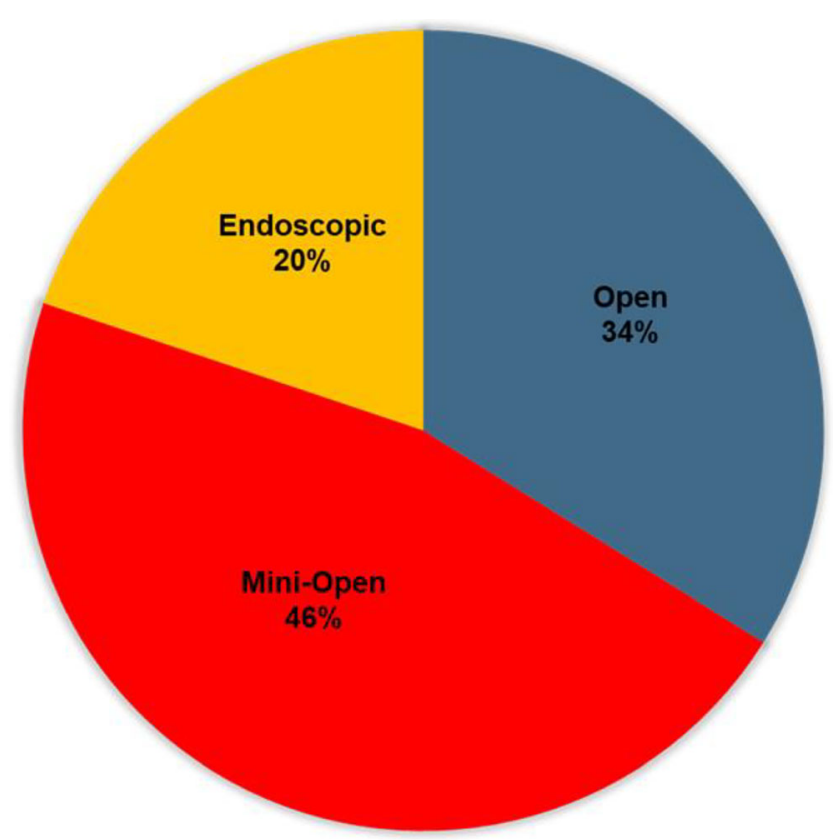

Fig. 1 According to a recent survey of the members of the American Association for Hand Surgery, only $20 \%$ of respondents utilize endoscopic techniques to divide the transverse carpal ligament in carpal tunnel surgery

\section{Return to Work}

Carpal tunnel syndrome is one of the most common disabling workplace injuries, accounting for $1-3$ cases per 1000 subjects per year. According to the United States Bureau of Labor Statistics, there was a median loss of 27 workdays with each claim in 1999 [16]. Kho et al [17•] evaluated a worker's compensation population and found that $89 \%$ of all patients returned to full duty work following carpal tunnel surgery. The average duration before returning to work was 12.5 weeks with a standard deviation of 11 weeks. The authors concluded that the permanent failure of $11 \%$ of CTS claimants to return to work imparts a significant socioeconomic cost to society and underscores the importance of returning these patients to work as quickly as possible.

Several studies have comparatively analyzed the ability of patients to return to work following surgery. In 2008, the American Academy of Orthopedic Surgeons (AAOS) published evidence-based guidelines for treatment of CTS. In its report, the AAOS examined pain outcomes, functional status, symptom severity, complications, and return to work following OCTR and ECTR. From this meta-analysis, 10 studies were identified which specifically addressed return to work. Three of these studies favored ECTR in returning patients back to work more quickly [18].

In a Cochrane Collaboration meta-analysis, 14 of 20 total studies evaluated return to work following open and endoscopic surgery. Eight studies found that patients undergoing ECTR were able to return to work more quickly, while only one study favored OCTR. A weighted mean difference was calculated and found that the time to return to work was 6 days earlier in patients undergoing endoscopic surgery [19].

Finally, a randomized prospective trial compared outcomes of OCTR with ECTR found a statistically significant difference between the two treatment groups with respect to returning to work. On average, patients in the endoscopic group returned to work 8 days sooner than patients undergoing open surgery. Based on these findings, the authors of the study recommended that "endoscopic carpal tunnel release be considered in the employed as a cost-effective procedure." [20]

\section{Functional Outcomes}

The superior results may be explained by the excellent functional outcomes imparted by endoscopic carpal tunnel surgery. In most of the literature, grip strength, pinch strength, and selfreported questionnaire outcomes have been used as a proxy for determining functional status. In a prospective randomized multi-center study evaluating 192 surgeries, patients undergoing ECTR demonstrated significantly greater grip strength, pinch strength, and hand dexterity compared with OCTR within the first 3 months after surgery. Scar sensitivity was 

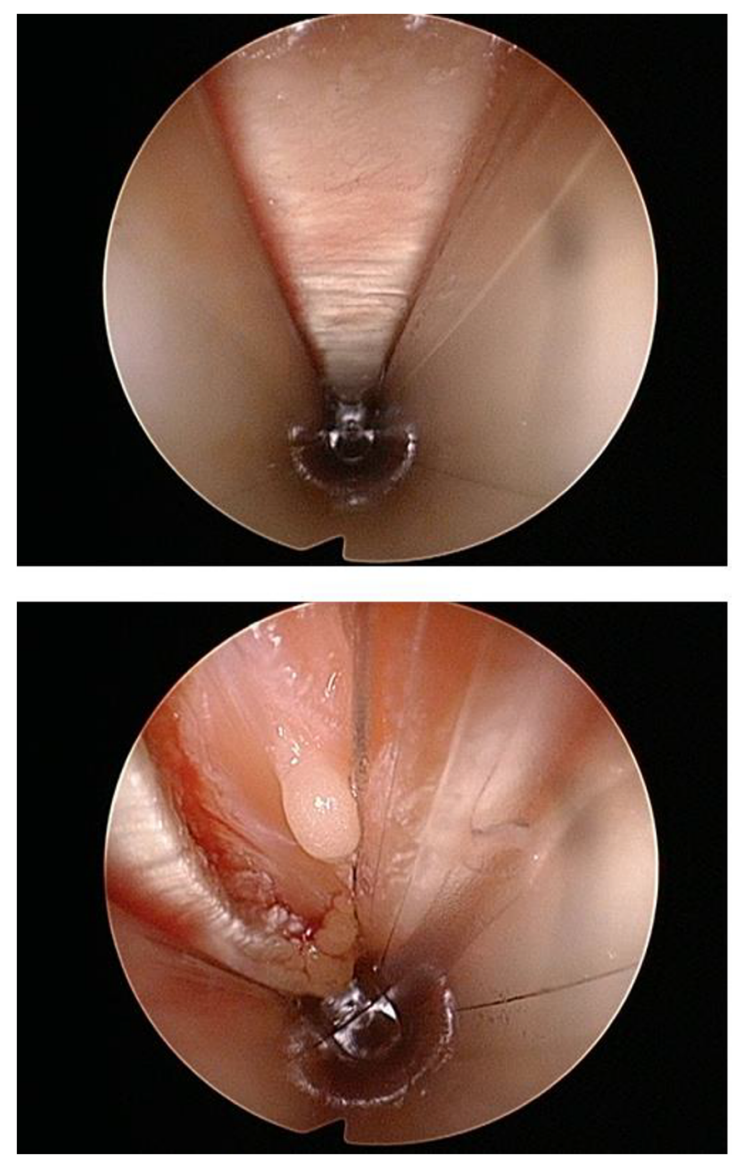

Fig. 2 a In endoscopic carpal tunnel surgery, the transverse fibers of the transverse carpal ligament must be clearly visualized prior to its division. b The endoscopic knife blade can then be deployed to safely cut the ligament. c Intraoperative photograph of the cut fibers using the

also superior compared with the OCTR group, though these findings equalized after 3 months. Carpal tunnel syndrome symptom severity scores, carpal tunnel syndrome functional status scores, and subjective satisfaction scores were reported as being superior in the ECTR group. The authors concluded that "good clinical outcomes and patient satisfaction are achieved more quickly when the endoscopic method of carpal tunnel release is used." [21]

Similar findings have also been supported by other studies $[22,23]$. Even in elderly patients over 65 years of age, clinical outcomes were found to be excellent with $79 \%$ of patients reporting that they were satisfied or very satisfied with their postoperative results. All 70 patients who presented with pain pre-operatively reported complete relief by the 6-month follow-up evaluation. The authors suggested that advanced symptoms and age at presentation should not be a contraindication to endoscopic carpal tunnel approaches [23].

Finally, sleep disturbance is a common complaint of patients with CTS. Patients consistently report nighttime symptoms include multiple awakenings due to hand pain and numbness. It is thought that wrist malposition during sleep can acutely exacerbate CTS symptoms by increasing pressure

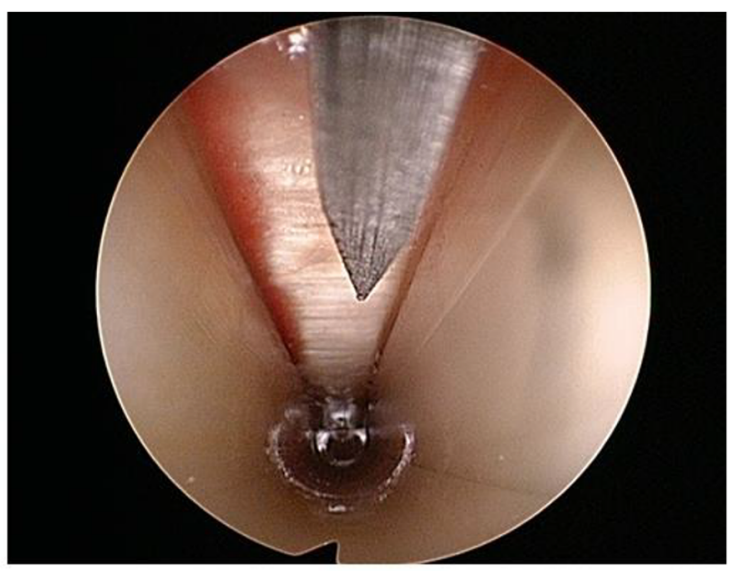

SafeView Soft Tissue Release System from Mission Surgical Innovations (Wayne, PA). The cannula is slightly rotated to see each cut end of the ligament

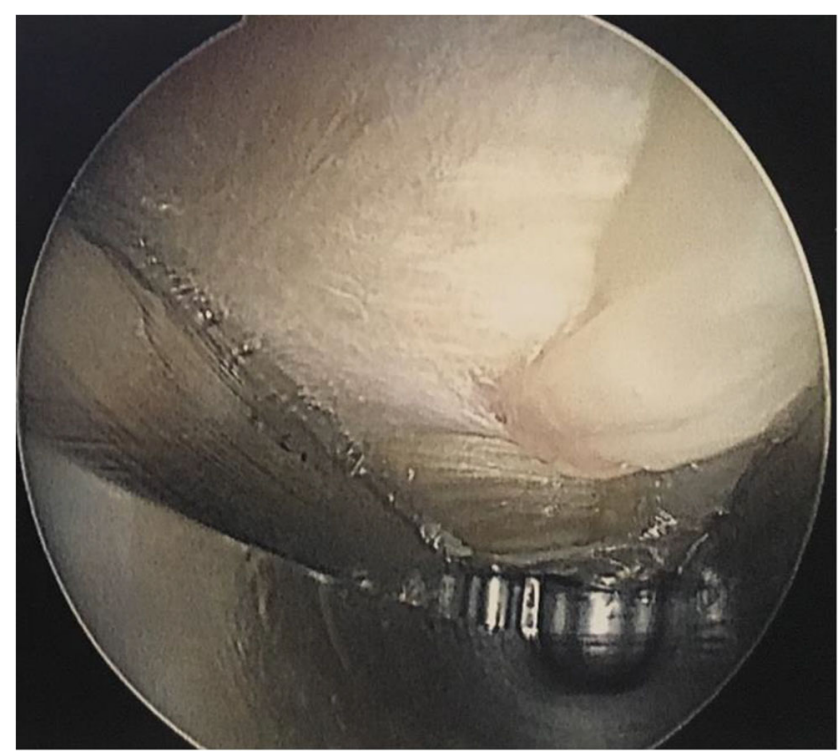

Fig. 3 With endoscopic techniques, unusual or abnormal anatomy can be visualized. The recurrent motor branch of the median nerve is shown here while the endoscope is directed palmarly toward the transverse carpal ligament using the synchronized endoscopic guide system by SegWay Orthopaedics (Carlsbad, CA) 
within the carpal canal [24]. For many patients, nighttime symptoms and subsequent daytime dysfunction are a strong impetus to seek medical care. A prospective comparison was performed in 60 patients, randomized to undergo either OCTR or ECTR surgery. Prior to surgery, patients were administered three self-reported outcome measures: the Pittsburgh Sleep Quality Index (PSQI), the Insomnia Severity Scale (ISI), and the Quick Disabilities of the Arm, Shoulder and Hand (QuickDASH) survey, which were subsequently administered at various postoperative time points. In this study, all 60 patients reported significant improvements in the three outcome scores by their first postoperative visit. ECTR provided superior improvement to OCTR at the first postoperative visit for ISI $(p=0.006)$ and PSQI $(p=0.016)$, and at the second visit for PSQI $(p=0.0038)$. There were no significant differences between the two groups for the QuickDASH at any time points. Therefore, endoscopic and open carpal tunnel surgeries both improve sleep symptoms postoperatively in the shortterm, though ECTR does so more rapidly [25].

\section{Complications}

While much of the initial skepticism surrounding endoscopic carpal tunnel surgery has waned, many still consider endoscopic approaches to be dangerous secondary to limited visualization and concern for nerve/arterial injury. In contrast, these concerns have not been strongly supported by the hand surgery literature. In a meta-analysis evaluating complications in 22,327 endoscopic cases and 5669 open cases, the incidence of structural damage to nerves, arteries, or tendons was found to be $0.49 \%$ in OCTR and $0.19 \%$ in ECTR. The difference was found to be statistically significant. Interestingly, transient neuropraxias were more common with ECTR, reported in 1.45\% of ECTR cases compared with $0.25 \%$ of OCTR surgeries. Arterial injuries occurred in only $0.02 \%$ of all patients in the endoscopic group. Therefore, selection of an open versus an endoscopic approach based on presumed structural complications or soft tissue injuries is not supported by statistical analysis of published complications [26].

Another study found that transient nerve problems were more frequently encountered with endoscopic surgery. In this meta-analysis, wound problems - such as infection, hypertrophic scar, and scar tenderness - were found to be more prevalent with open approaches in seven randomized trials comparing OCTR with ECTR [27]. Despite the slightly increased incidence of transient neuropraxias with ECTR, complex regional pain syndrome (CRPS) appears to occur with the same relative frequency regardless of the surgical approach. In a retrospective review, rates of CRPS were nearly identical between surgery types for both privately insured $(0.3 \%)$ and Medicare $(0.1 \%)$ patients. Middle-aged and female patients had significantly higher rates of CRPS than did the general population. The decision, therefore, between OCTR and
ECTR should not be made from concern for development of CRPS, as the incidence was found to be low and similar for both approaches [28].

A recent study found that endoscopic carpal tunnel surgery was associated with fewer overall complications than open carpal tunnel surgery in the Medicare and private insurance patient populations. A significantly lower percentage of patients in the ECTR group experienced a postoperative infection and wound dehiscence than in the OCTR group. In the Medicare population, median nerve injury occurred in 0.59 / 1000 ECTRs versus 1.69/1000 OCTRs [29•].

In sum, these studies demonstrate that major irreversible nerve injury is rare with carpal tunnel surgery and that endoscopic approaches are safe compared with open surgery. It appears there is an increased risk of reversible nerve injuries or neuropraxia with ECTR, while there is an increased risk of wound complications with OCTR. The increased risk of neuropraxia in ECTR is most likely related to median nerve irritation or increased carpal canal pressure induced by insertion of a cannula or endoscope. Wound issues with OCTR can probably be explained by the location of the incision at the base of the hand between the thenar and hypothenar eminences. Early motion may cause tension at the suture line, thereby increasing the risk of infection or wound dehiscence.

\section{Cost}

While endoscopic surgery is seemingly more expensive, a cost analysis comparing endoscopic and open carpal tunnel surgery found that the incremental cost difference was $\$ 46$ in favor of endoscopic techniques, using Medicare costs and probabilities of various outcomes [30].

A 2002 study found that the mean direct cost was $\$ 3750$ for endoscopic carpal tunnel surgery, compared with $\$ 3940$ for open carpal tunnel surgery. These direct costs included surgeon fees, anesthesia fees, and capital expenditures. The lower cost of ECTR was attributed to the increased operating room time OCTR may require in this multi-center study. The authors concluded that endoscopic carpal tunnel surgery imparts a lower indirect cost to society and is especially valuable given the faster return to work these patients demonstrated [21].

These findings contradict more recent data suggesting greater expenditures for endoscopic approaches. A 2019 retrospective review found that ECTR costs more than OCTR for both the Medicare populations ( $\$ 1643$ versus $\$ 1015$ per procedure, $p<0.001)$ and privately insured patients $(\$ 1928$ versus $\$ 1191$ per procedure, $p<0.001$ ) [29•].

In another study, time-driven activity-based costing (TDABC) was used to characterize procedural costs of OCTR and ECTR. Total costs were calculated by aggregating individual resource utilization and time data and were compared between the two surgical approaches. Through TDABC 
methodology, the authors found that the total procedural cost for ECTR was $43.9 \%$ greater than OCTR (\$2760 versus $\$ 1918$ ) at an academic medical center employing resident trainees. The cost differences were largely driven by disposable endoscopic blade assembly (\$217), direct operating room costs related to procedural duration (44.8 versus $40.5 \mathrm{mi}-$ nutes), and physician labor [31•].

\section{Discussion}

In summary, carpal tunnel surgery is effective with few complications. Though patient outcomes are often similar or even slightly superior with endoscopic carpal tunnel surgery when compared with open approaches, patients express significantly greater satisfaction with minimally invasive endoscopic surgery.

A prospective randomized evaluation of OCTR and ECTR found that both techniques were "well tolerated with no differences in functional outcomes ... and complications." In these patients, the operative approach was randomly assigned in one hand. Approximately 1 month later, the alternative technique was performed on the contralateral side. There were no differences in pain scores, two-point discrimination, monofilament testing, or grip strength at any postoperative time point. However, overall satisfaction was significantly greater in the ECTR group. The authors concluded, "Although there were no differences between the two groups, patients still preferred the ECTR, demonstrated by significantly higher overall satisfaction scores at the conclusion of the study." [32•]

With endoscopic carpal tunnel surgery, I offer my patients improved postoperative discomfort and the potential for earlier return to work, balanced by the increased risk of transient neuropraxia. Though the early advantages of endoscopic carpal tunnel surgery appear to be erased by 3 months, patients express greater enthusiasm for the minimally invasive approach. Appropriate patient selection is important for successful outcomes. Patients with known anatomic anomalies, fracture history, previous CTS surgery, or inflammatory tenosynovitis are probably best served with an open or mini-open approach. If inadequate visualization is encountered during surgery, my threshold to abort endoscopic surgery and to convert to a mini-open incision is low. Complications are more likely to occur where endoscopic surgery is doggedly pursued with inappropriate visualization of the transverse carpal ligament. The transverse fibers on the undersurface of the ligament should be clearly seen prior to its division (Fig. 2).

My personal experience is that endoscopic carpal tunnel surgery is largely driven by patient's demands and expectations. Concerns over catastrophic complications appear to be overstated, and identification of abnormal or aberrant anatomy is still possible with endoscopic approaches (Fig. 3). From the surgical literature, there is no true consensus on surgical outcomes given the heterogeneity of study designs and techniques.

\section{Compliance with Ethical Standards}

Conflict of Interest Eon K. Shin declares that he owns stock and intellectual property in Mission Surgical Innovations, serves on the scientific advisory board for In2Bones, and receives consultation payments from DePuy Synthes.

Human and Animal Rights and Informed Consent This article does not contain any studies with human or animal subjects performed by any of the authors.

\section{References}

Papers of particular interest, published recently, have been highlighted as:

\section{- Of importance}

1. Botte MJ, von Schroeder HP, Abrams RA, Gellman H. Recurrent carpal tunnel syndrome. Hand Clin. 1996;12(4):731-43.

2. Learmonth JR. The principle of decompression in the treatment of certain diseases of peripheral nerves. Surg Clin North Am. 1933;13: 905-13.

3. Cannon BW, Love JG. Tardy median palsy; median neuritis; median thenar neuritis amenable to surgery. Surgery. 1946;20:210-6.

4. Brain WR, Wright AD, Wilkinson M. Spontaneous compression of both median nerves in the carpal tunnel. Lancet. 1947;1:277-82.

5. Phalen GS, Gardner WJ, La Londe AA. Neuropathy of the median nerve due to compression beneath the transverse carpal ligament. J Bone Joint Surg Am. 1950;32:109-12.

6. Phalen GS. Spontaneous compression of the median nerve at the wrist. JAMA. 1951;145:1128-33.

7. Phalen GS, Kendrick JI. Compression neuropathy of the median nerve in the carpal tunnel. JAMA. 1957;164:524-30.

8. Phalen GS. The carpal tunnel syndrome. Seventeen years' experience in diagnosis and treatment of six hundred fifty-four hands. $\mathrm{J}$ Bone Joint Surg Am. 1966;48:211-28.

9. Phalen GS. Reflections on 21 years' experience with the carpaltunnel syndrome. JAMA. 1970;212:1365-7.

10. Phalen GS. The carpal tunnel syndrome. Clinical evaluation of 598 hands. Clin Orthop. 1972;83:29-40.

11. Okutsu I, Ninomiya S, Natsuyama M, Takatori Y, Inanami H, Kuroshima N, et al. Subcutaneous operation and examination under universal endoscope. Nihon Seikeigeka Gakkai Zasshi. 1987;61: 491-8 [Jpn].

12. Chow JC. Endoscopic release of the carpal ligament: a new technique for carpal tunnel syndrome. Arthroscopy. 1989;5:19-24.

13. Feinstein P. Endoscopic carpal tunnel release in a community-based series. J Hand Surg Am. 1993;18(3):451-4.

14. Shin EK, Bachoura A, Jacoby SM, Chen NC, Osterman AL. Treatment of carpal tunnel syndrome by members of the American Association for Hand Surgery. Hand. 2012;7(4):351-6.

15. American Academy of Orthopedic Surgeons. Management of carpal tunnel syndrome: evidence-based clinical practice guideline. Published 2016. www.aaos.org/ctsguideline. Accessed 20 Aug 2019.

16. United States Department of Labor Bureau of Labor Statistics. Days away from work highest for carpal tunnel syndrome. https://www. 
bls.gov/opub/ted/2001/apr/wk1/art01.htm. Published 2001. Accessed 20 Aug 2019.

17. Kho JY, Gaspar MP, Kane PM, Jacoby SM, Shin EK. Prognostic variables for patient return-to-work interval following carpal tunnel release in a workers' compensation population. Hand. 2017;12(3): 246-51 The purpose of this manuscript was to determine factors that contribute to time to return to work in workers' compensation patients who have undergone carpal tunnel surgery. The average return-to-work time in this study was 12.5 weeks. Psychiatric diagnoses were the most predictive factors for delay in returning to work, followed by opioid use, chronic pain conditions, and pre-operative work status. There was no association between return-to-work and marital status, body mass index, and preoperative electrodiagnostic values.

18. American Academy of Orthopedic Surgeons. Clinical practice guideline on the treatment of carpal tunnel syndrome. Published 2008. https://www.aaos.org/research/guidelines/CTSTreatmentGuideline.pdf. Accessed 20 Aug 2019.

19. Scholten RJ, van der Mink MA, Uitdehaag BM, Bouter LM, de Vet HC. Surgical treatment options for carpal tunnel syndrome. Cochrane Database Syst Rev. 2007;17(4):CD003905.

20. Saw NL, Jones S, Shepstone L, Meyer M, Chapman PG, Logan AM. Early outcome and cost-effectiveness of endoscopic versus open carpal tunnel release: a randomized prospective trial. J Hand Surg Br. 2003;28(5):444-9.

21. Trumble TE, Diao E, Abrams RA, Gilbert-Anderson MM. Singleportal endoscopic carpal tunnel release compared with open release: a prospective, randomized trial. J Bone Joint Surg Am. 2002;84(7): $1107-15$

22. Abrams R. Endoscopic versus open carpal tunnel release. J Hand Surg Am. 2009;34(3):535-9.

23. Beck JD, Wingert NC, Rutter MR, Irgit KS, Tang X, Klena JC. Clinical outcomes of endoscopic carpal tunnel release in patients 65 and over. J Hand Surg Am. 2013;38(8):1524-9.

24. McCabe SJ, Uebele AL, Pihur V, Rosales RS, Atroshi I. Epidemiologic associations of carpal tunnel syndrome and sleep position: is there a case for causation? Hand. 2007;2(3):127-34.

25. Gaspar MP, Osterman MN, Shin EK, Osterman AL, Kane PM. Sleep disturbance and response to surgical decompression in patients with carpal tunnel syndrome: a prospective randomized pilot comparison of open versus endoscopic release. Acta Biomed. 2019;90(1):92-6.

26. Benson LS, Bare AA, Nagle DJ, Harder VS, Williams CS, Visotsky JL. Complications of endoscopic and open carpal tunnel release. Arthroscopy. 2006;22(9):919-24.

27. Gerritsen AA, Uitdehaag BM, van Geldere D, Scholten RJ, de Vet HC, Bouter LM. Systematic review of randomized clinical trials of surgical treatment for carpal tunnel syndrome. Br J Surg. 2001;88(10):1285-95.
28. Mertz K, Trunzter J, Wu E, Barnes J, Eppler SL, Kamal RN. National trends in the diagnosis of CRPS after open and endoscopic carpal tunnel release. J Wrist Surg. 2019;8(3):209-14.

29. Devana SK, Jensen AR, Yamaguchi KT, D’Oro A, Buser Z, Wang $\mathrm{JC}$, et al. Trends and complications in open versus endoscopic carpal tunnel release in private payer and Medicare patient populations. Hand. 2019;14(4):455-61 This study compared complications and costs associated with endoscopic carpal tunnel release and open carpal tunnel release. A significantly lower percentage of patients in the endoscopic group experienced postoperative infection and wound dehiscence. Median nerve injury occurred less frequently in the endoscopic group compared with the open group for both Medicare and private payer health insurance populations. However, endoscopic surgery was more costly than open surgery.

30. Chung KC, Walters MR, Greenfield ML, Chernew ME. Endoscopic versus open carpal tunnel release: a costeffectiveness analysis. Plast Reconstr Surg. 1998;102(4):1089-99.

31. Koehler DM, Balakrishnan R, Lawler EA, Shah AS. Endoscopic versus open carpal tunnel release: a detailed analysis using timedriven activity-based costing at an academic medical center. J Hand Surg Am. 2019;44(1):e1-62.e9 In this study, the authors used time-driven activity-based costing (TDABC), a novel accounting technique, to determine procedural costs of endoscopic versus open carpal tunnel surgery. In academic medical centers employing resident trainees, the total procedural cost for endoscopic surgery was found to be $43.9 \%$ greater than the open approach. The cost difference was primarily driven by the use of a disposable endoscopic blade, direct operating room costs related to procedural duration, and physician labor.

32. Michelotti B, Romanowsky D, Hauck RM. Prospective, randomized evaluation of endoscopic versus open carpal tunnel release in bilateral carpal tunnel syndrome: an interim analysis. Ann Plast Surg. 2014;73(Suppl 2):S157-60 This manuscript explored patient satisfaction with endoscopic carpal tunnel surgery compared with open carpal tunnel surgery. The two cohorts exhibited equivalent pain scores, two-point discrimination, monofilament testing, thenar strength, and overall grip strength at all postoperative time points. However, overall satisfaction scores were significantly higher in the endoscopic group compared with the open group. No complications were recorded in either group. The authors concluded, "Although there were no differences between the groups using our study metrics, patients still preferred endoscopic carpal tunnel surgery, demonstrated by significantly higher overall satisfaction scores at the conclusion of the study.".

Publisher's note Springer Nature remains neutral with regard to jurisdictional claims in published maps and institutional affiliations. 